\title{
Experiência baseada em Gamificação no Ensino sobre Herança em Programação Orientada a Objetos
}

\author{
Janderson Jason Barbosa Aguiar ${ }^{1}$ \\ ${ }^{1}$ Universidade Federal de Campina Grande (UFCG), Campina Grande - PB - Brasil \\ janderson@copin.ufcg.edu.br
}

\begin{abstract}
Motivate students during the teaching of programming is still often a challenge. The gamification can be used for this motivation. The purpose of this paper is to present a gamification experience as a strategy for the teaching-learning process concerning the concept of inheritance in ObjectOriented Programming (OOP), with students of computer science at technical level. With this experience, it was possible to identify a more collaborative learning environment and, in general, greater engagement of the students during the class. It is hoped that this paper can encourage the use of collaborative and competitive aspects aiming at the learning of OOP and programming in general.
\end{abstract}

Resumo. Motivar alunos durante o ensino de programação ainda é muitas vezes um desafio. A gamificação pode ser utilizada para propiciar essa motivação. $O$ objetivo deste artigo consiste em apresentar um relato de experiência baseada em gamificação como estratégia para o processo de ensino-aprendizagem relativo ao conceito de Herança em Programação Orientada a Objetos (POO), no âmbito de um curso técnico em informática. Com esta experiência, foi possivel identificar um ambiente mais colaborativo de aprendizagem e, em geral, maior engajamento dos alunos da turma durante a aula. Espera-se com este artigo incentivar a utilização de aspectos colaborativos e competitivos, objetivando o aprendizado de temas do paradigma $\mathrm{POO}$ e programação em geral.

\section{Considerações Iniciais}

É desafiador, para o professor, em qualquer nível de ensino, tornar o processo de ensino-aprendizagem mais interessante [Haetinger e Graziola Junior 2002].

Em se tratando de disciplinas de programação, são conhecidas pelos professores as dificuldades que os alunos enfrentam inicialmente para compreender os aspectos para construir programas (domínio de estruturas condicionais, de repetição etc.) e, depois que os alunos alcançam o mínimo de conhecimento necessário, eles geralmente enfrentam nos semestres consecutivos a quebra de paradigma da programação estruturada para a Programação Orientada a Objetos (POO) [Vahldick 2007].

Segundo Zaina e Caversan (2005), quando o aluno inicia o contato com uma linguagem orientada a objetos, há muitas vezes dificuldade em cruzar as barreiras que diferenciam os estilos de POO e do paradigma de programação imperativa.

É interessante reforçar a existência dessas dificuldades em cursos técnicos em informática, nos quais muitos alunos - que geralmente preferem aspectos envolvendo manutenção e redes de computadores -, quando se deparam com disciplinas 
envolvendo programação, apresentam pouca motivação em programar computadores e/ou pouco vislumbram a necessidade de se estudar tais conceitos.

$\mathrm{O}$ ambiente escolar precisa dar prioridade à busca pela concepção de espaços de aprendizagem e experimentação utilizando tecnologias que permitam aos alunos serem livres para criar, questionar e aprender entre si [Scaico e De Queiroz 2013] [Da Silva e De Queiroz 2014]. Nesse sentido, é cada vez mais frequente o uso de jogos instrutivos no espaço escolar, uma vez que despertam "uma necessidade convincente para saber, uma necessidade de perguntar, examinar, assimilar e dominar certas habilidades e áreas de conteúdo" [Da Silva e De Queiroz 2014].

O termo Gamificação — oriundo da palavra inglesa Gamification — consiste no uso de características e princípios de jogos a fim de facilitar o aprendizado e a resolução de problemas [Paschoal et al. 2014].

\begin{abstract}
"Alguns especialistas argumentam que jogos são, antes de qualquer coisa, sistemas de aprendizagem, e que isso dá conta do sentido de engajamento e entretenimento que os jogadores experimentam." [Da Silva e De Queiroz 2014]
\end{abstract}

A gamificação oferece oportunidades para motivar e engajar os alunos [Lee e Hammer 2011] [Kapp 2012]. Quando aplicada à educação, a gamificação tem como principal objetivo utilizar os mecanismos de jogos na concepção de iniciativas educativas, tentando tornar os conteúdos mais atrativos [Domínguez et al. 2013] [Seixas et al. 2014].

Para fazer uso da gamificação, segundo Liu, Alexandrova e Nakajima (2011 apud França e Reategui 2013), inicia-se a interação com base em um objetivo/desafio e em uma condição de vitória, sendo dadas recompensas quando o usuário alcança uma determinada meta - geralmente uma pontuação, moeda virtual etc. —, e, para motivar a colaboração e a competição, são fornecidos aos participantes emblemas e/ou um quadro de líderes baseados no sistema de pontos.

O Duolingo (https://www.duolingo.com) e o Livemocha (http://livemocha.com) são exemplos de ambientes on-line que possuem elementos de gamificação, ambos voltados ao processo de ensino-aprendizagem de idiomas.

Neste artigo, é apresentado um relato de experiência baseada em gamificação, considerando uma abordagem competitiva e colaborativa, no ensino de programação (conceito de Herança em POO) para alunos de um curso técnico em informática. O restante do artigo está estruturado da seguinte maneira: na Seção 2, são comentados trabalhos relacionados; na Seção 3, são explanadas as características dessa experiência de gamificação; na Seção 4, são discutidos resultados; e, na Seção 5, são apresentados alguns comentários finais.

\title{
2. Trabalhos Relacionados
}

Com o objetivo de tornar o processo de ensino-aprendizagem mais dinâmico, Haetinger e Graziola Junior (2002) propuseram o uso de um robô como ferramenta de apoio à aprendizagem do conceito de orientação a objetos. Os autores observaram que a atividade prática desenvolvida foi um diferencial importante, aumentando o interesse e a motivação pela aula por parte dos graduandos. 


\section{CBIE-LACLO 2015}

Anais dos Workshops do IV Congresso Brasileiro de Informática na Educação (CBIE 2015)

Vahldick (2007) realizou uma experiência, enquanto professor da disciplina de Programação Orientada a Objetos em Java, evidenciando o uso de metáforas, da interação com objetos e da escolha de um ambiente de programação para favorecer o lúdico no aprendizado. Apesar dos resultados não motivadores (índices de aprovação entre $30 \%$ e $40 \%$ ), o autor afirma que a qualidade do código final dos aprovados foi superior à qualidade de turmas anteriores.

Considerando a similaridade entre os pensamentos de filósofos e a Orientação a Objetos, Chaves et al. (2010) apresentaram uma abordagem de ensino interdisciplinar, utilizando a Teoria das Ideias de Platão e a Teoria da Forma e Substância de Aristóteles para fundamentar o ensino dos conceitos de POO. Os resultados indicaram que todos os alunos concordaram que a Teoria de Platão e Aristóteles poderiam ser introdutórias à POO e, apesar da maioria dos alunos inicialmente apresentar resistência à abordagem, todos os alunos indicaram, ao final da disciplina, que a analogia feita tornou o assunto mais interessante. Além disso, todos os alunos concordaram que a abordagem realizada deve ser estendida para outras disciplinas.

Em relação à gamificação aplicada à educação, há vários trabalhos na literatura. Borges et al. (2013) realizaram um mapeamento sistemático com o objetivo de colaborar com uma visão geral sobre a área. Os resultados apontaram que os estudos se concentraram, em sua maioria, na investigação de como a gamificação pode ser usada para motivar, aprimorar habilidades e maximizar o aprendizado dos alunos.

Fernandes e Castro (2013) apresentaram um ambiente desenvolvido para ensino de química orgânica, baseado em gamificação, testado e aprovado por professores, além de ter sido considerado interessante pelos alunos. Medeiros, Fernandes e Damasceno (2014) apresentaram uma proposta de jogo digital, no formato de RPG (Role Playing Game), como forma alternativa auxiliar na campanha de prevenção ao uso de drogas.

Seixas et al. (2014) utilizaram a gamificação como estratégia no engajamento de alunos do $9^{\circ}$ ano do ensino fundamental, sendo utilizadas duas plataformas Web para realizar a mecânica de recompensa. Os resultados indicaram que os alunos que obtiveram as melhores médias foram os que receberam mais recompensas do professor.

Alves et al. (2014) desenvolveram um jogo educativo, denominado ControlHarvest, para explorar aspectos da ecologia das populações, as funções do controle biológico e as especificidades de algumas relações ecológicas. O jogo foi avaliado por 14 alunos do ensino médio/técnico integrado, que indicaram interesse a respeito do conteúdo abordado pelo ControlHarvest.

Figueiredo (2015) apresentou uma proposta, em andamento, para a utilização de gamificação em disciplinas de um curso de Sistemas de Informação (Algoritmos III e Projeto de Sistemas I) visando ao engajamento e à motivação dos alunos. Segundo a autora, os jogos, até o momento, mostraram-se efetivos para o aumento da participação dos alunos em sala, além de maior comprometimento com a realização de atividades.

Apesar dos trabalhos citados nesta seção estarem relacionados ao trabalho descrito neste artigo, eles diferenciam-se essencialmente por não serem experiências de gamificação, considerando o ensino sobre herança (um dos conceitos essenciais em POO [Vahldick 2007]), com alunos de nível técnico. 
CBIE-LACLO 2015

Anais dos Workshops do IV Congresso Brasileiro de Informática na Educação (CBIE 2015)

\section{Metodologia}

A estratégia baseada em gamificação para abordar o conceito de Herança em POO foi empregada no Curso Técnico em Informática da Escola Técnica Redentorista (Campina Grande - PB) — técnico de nível médio —, com 30 alunos, cuja base tecnológica (disciplina) é denominada Desenvolvimento de Aplicativos com Banco de Dados (DABD), na qual, entre os conceitos abordados, abrange POO por meio da linguagem de programação Java.

A experiência consistiu na realização de uma atividade que visou a verificação da aprendizagem de conteúdos abordados em aulas anteriores (especialmente sobre herança). Destaca-se que a atividade contemplou também os conteúdos abordados desde o início da disciplina, uma vez que os alunos precisavam de conhecimento do ambiente Eclipse, além de conhecer as características básicas da linguagem Java para solucionar as etapas finais de cada problema.

A turma foi dividida em 5 grupos (6 alunos, em média, por grupo) sendo utilizados essencialmente como recursos um computador (configurado para executar Java, por meio do ambiente Eclipse, e para apresentar os slides com os enunciados dos problemas a serem resolvidos), um datashow (para projetar as regras, os enunciados dos problemas e a implementação das soluções por parte dos alunos) e a lousa da sala de aula (para resolução da primeira etapa de cada problema).

Foram elaborados 5 problemas, um para cada grupo, além de um Problema Extra para o caso de empate ao final da atividade (ver Quadro 1). Os problemas foram definidos sequencialmente para os grupos: o Problema 1 destinado ao Grupo 1, o Problema 2 destinado ao Grupo 2, e assim sucessivamente. Os grupos, por sua vez, foram definidos aleatoriamente.

\section{Quadro 1. Problemas Elaborados para Atividade sobre Herança.}

Problema 1: Represente um minimundo de funcionários em uma empresa de TI. Os Programadores têm matrícula, nome e domínio em uma linguagem de programação, além de saberem programar. Os Especialistas em Rede também têm matrícula e nome, mas estes gerenciam as redes da empresa.

Problema 2: Represente um minimundo com mensagens de SMS e do Whatsapp. Considere que todas elas têm um conteúdo, o nome do remetente e o número do celular do destinatário. Entretanto, para mensagens do Whatsapp, é preciso verificar se há conexão com a Internet.

Problema 3: Represente um minimundo de Pessoas Físicas e Pessoas Jurídicas. Considere que todas as pessoas têm nome, idade e podem trabalhar. Entretanto, Pessoas Físicas têm CPF enquanto Pessoas Jurídicas têm CNPJ e podem abrir uma empresa.

Problema 4: Represente um minimundo de Navios, Carros, Submarinos e Motocicletas. Todos são Meios de Transporte e possuem uma quantidade de passageiros. Os meios Marítimos podem ser submersos ou não. As motocicletas, apesar de serem um meio Terrestre como os Carros, ao serem criadas, deve-se deixar claro que a quantidade de passageiros é 2 .

Problema 5: Represente um minimundo para criação de Pontos, Círculos e Cilindros. Um Ponto possui as coordenadas $\mathrm{X}$ e $\mathrm{Y}$. Um Círculo, além dessas coordenadas, possui um raio; e, além disso, pode-se calcular sua área. Um Cilindro, além das coordenadas, raio e cálculo da área, possui uma altura e pode-se calcular seu volume.

Problema Extra: Represente um minimundo de Professores e Estudantes. Sabe-se que ambos têm nome e matrícula. Entretanto, Professores ministram aulas e Alunos assistem às aulas. 


\section{CBIE-LACLO 2015}

Anais dos Workshops do IV Congresso Brasileiro de Informática na Educação (CBIE 2015)

Em cada problema, os grupos precisavam realizar estas 3 etapas: (1) a modelagem do problema em classes (utilizando conceitos simples de UML); (2) a implementação das classes em Java; e (3) alguns "testes" simples da implementação (instanciação e uso de objetos) em um método main. Vale ressaltar que, para a implementação dos métodos, foi sugerido apenas imprimir na tela (comando System.out.println em Java) alguma frase relativa à ação do método — com exceção do Problema 5, o último, que ficou definido que o grupo implementaria com a ajuda do professor.

Além da definição aleatória dos alunos integrantes de cada grupo, para cada problema os integrantes foram sorteados (sem repetição) para realizar cada uma das 3 etapas da atividade. Foi frisado que os demais integrantes do grupo poderiam (e deveriam!) ajudar, em cada etapa, o colega sorteado de seu grupo, ao mesmo tempo em que os outros grupos não poderiam atrapalhar (com barulho etc.).

Foi definido que cada etapa valeria 2 pontos, podendo um problema totalizar 6 pontos para o grupo. O professor ficou responsável por julgar a corretude das respostas, podendo considerar que a etapa valeu 2 pontos, 1 ou 0 ponto. Os outros grupos também puderam opinar no sentido de avaliar a corretude das respostas.

Uma regra definida foi que, se o grupo não fizesse determinada etapa, poderia indicar outro grupo a fazer; neste caso, os pontos destinados à etapa seriam divididos para ambos os grupos. Além disso, se o grupo indicado também não fizesse, qualquer outro grupo poderia fazer; neste caso, os pontos destinados à etapa seriam apenas para 0 grupo que se dispôs. A intenção dessas regras foi para que todas as etapas fossem realizadas pelos alunos, não pelo professor.

Foi comentado no início da experiência que outras regras seriam explicadas conforme necessidade. Além disso, foi comentado, como premiação, que o grupo com mais pontos ao final ganharia chocolates; e também foi frisado que o desempenho de cada um (participação/colaboração) fazia parte do processo de avaliação contínua do curso.

\section{Resultados e Discussão}

A experiência caracterizada na Seção 3 durou dois dias, com duas horas de aula em cada dia (totalizando 4 horas). Após a explicação inicial sobre a atividade, os grupos foram aleatoriamente definidos e não foi determinado um tempo limite para cada etapa, visando a uma liberdade maior para os alunos pensarem e apresentarem suas soluções.

É importante ressaltar que, embora não tenha sido determinado tempo limite, cada grupo - após a leitura do problema e escolha aleatória do representante do grupo para resolver uma etapa - precisou decidir, em pouco tempo (aproximadamente 1 minuto) se resolveria tal etapa ou usaria o recurso de indicar outro grupo para solucionála.

À medida que o grupo da vez terminava, diante da turma, a solução de determinada etapa, tal solução foi comentada pelo professor, havendo discussão com o restante dos grupos que opinavam sobre a solução. Não houve casos de discordância para a definição das pontuações $(2,1$ ou 0$)$. Nos casos em que a solução para a primeira ou para a segunda etapa não foi considerada completa, o professor, em discussão com a turma, complementou a solução para a etapa seguinte não ser comprometida. 
Durante a atividade, foram obtidas soluções, em geral, satisfatórias para todas as etapas. No Quadro 2 é apresentada uma dessas soluções. Ao final da atividade, não houve empate e não foi utilizado o Problema Extra.

\section{Quadro 2. Resposta do Grupo 1 para a Etapa 3 relativa ao Problema 1.}

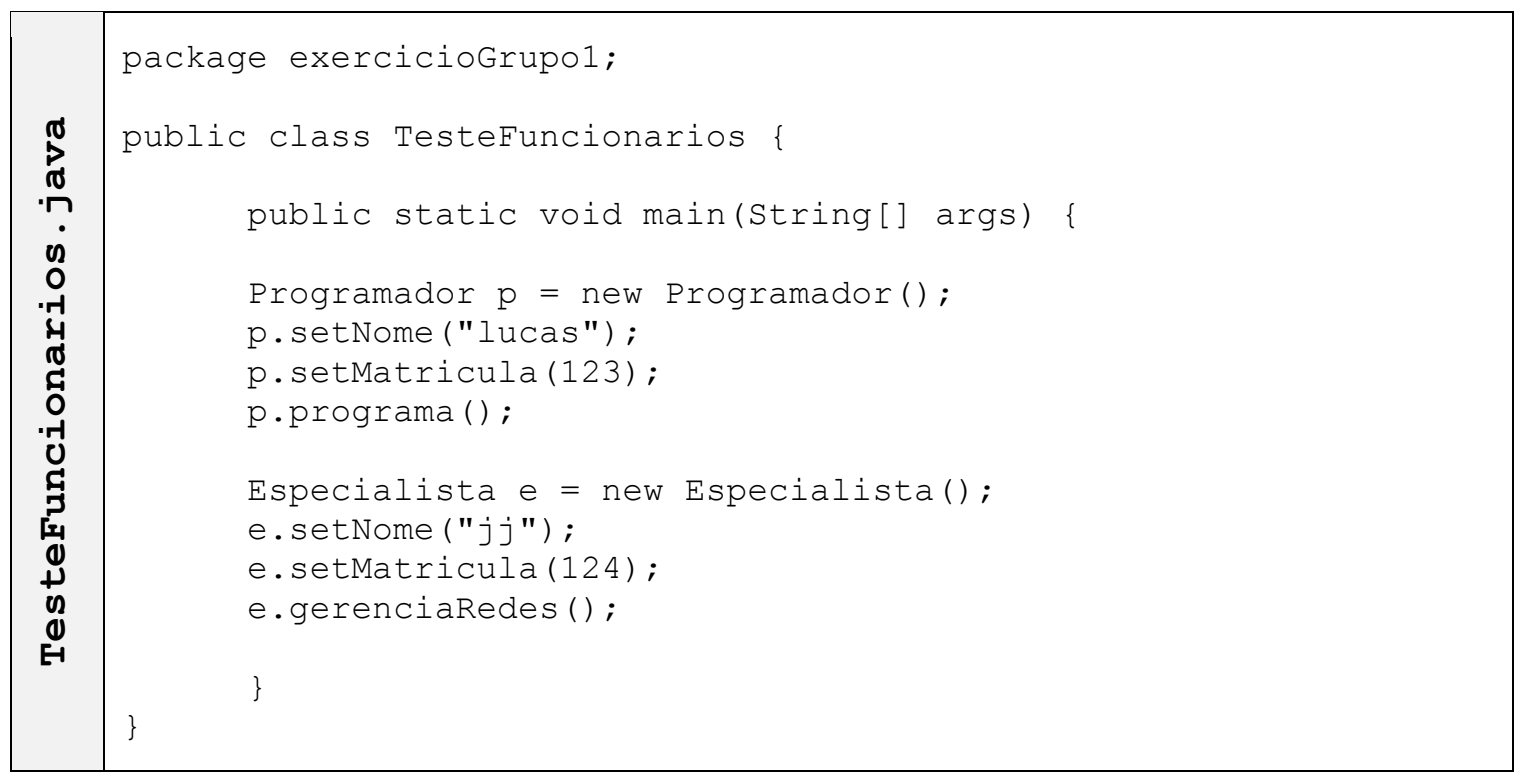

Com a realização da experiência, foi possível perceber, dentre vários aspectos, maior engajamento dos alunos com a disciplina. Muitos alunos da turma não se empolgavam em participar das aulas de DABD; entretanto, alunos totalmente calados em aulas tradicionais (alguns por timidez, outros por falta de interesse) participaram desta experiência, respondendo aos questionamentos. Um dos alunos, por exemplo, aproximou-se em particular para elogiar o professor, ao final da atividade, pela metodologia.

Durante a experiência, os alunos discutiram ideias em grupo para chegar a soluções e o professor pôde acompanhar a participação e interesse destes. Mesmo aqueles que apresentavam dificuldade na base tecnológica (disciplina) tentaram contribuir com ideias, entendendo o conceito de herança mesmo que tivessem dificuldades no aspecto da sintaxe em Java.

Todas as etapas de todos os problemas foram realizadas pelos alunos (não foi necessário o professor apresentar sozinho a solução), sendo utilizada algumas vezes a regra de outro grupo resolver alguma etapa do problema. Apenas no Problema 5, como citado anteriormente, o professor ajudou mais fortemente devido à implementação relativa a cálculos de área e volume de formas geométricas. Em relação à modelagem, embora foram utilizados conceitos de UML, não foi cobrado muito detalhe por parte dos alunos; todavia, o professor, sempre que julgou necessário, realizou comentários de como a modelagem poderia ser melhorada.

É necessário comentar que um dos grupos não teve uma participação tão efetiva quanto os outros - alguns alunos deste grupo não mostraram interesse durante a participação dos demais grupos e seus problemas. Todavia, na sua vez, tal grupo participou de maneira satisfatória. 
CBIE-LACLO 2015

Anais dos Workshops do IV Congresso Brasileiro de Informática na Educação (CBIE 2015)

Apesar da atividade ser colaborativa, o professor avaliou cada aluno também de maneira individual. Na Figura 1 é apresentado o desempenho da turma nas atividades da disciplina DABD realizadas até a experiência comentada neste artigo (Atividade 5). As atividades 1 a 4 foram, em geral, resumos e resoluções de exercícios tradicionais de programação. Em vez de notas, as atividades foram avaliadas por conceitos - a saber: Insuficiente, Regular, Bom e Ótimo.

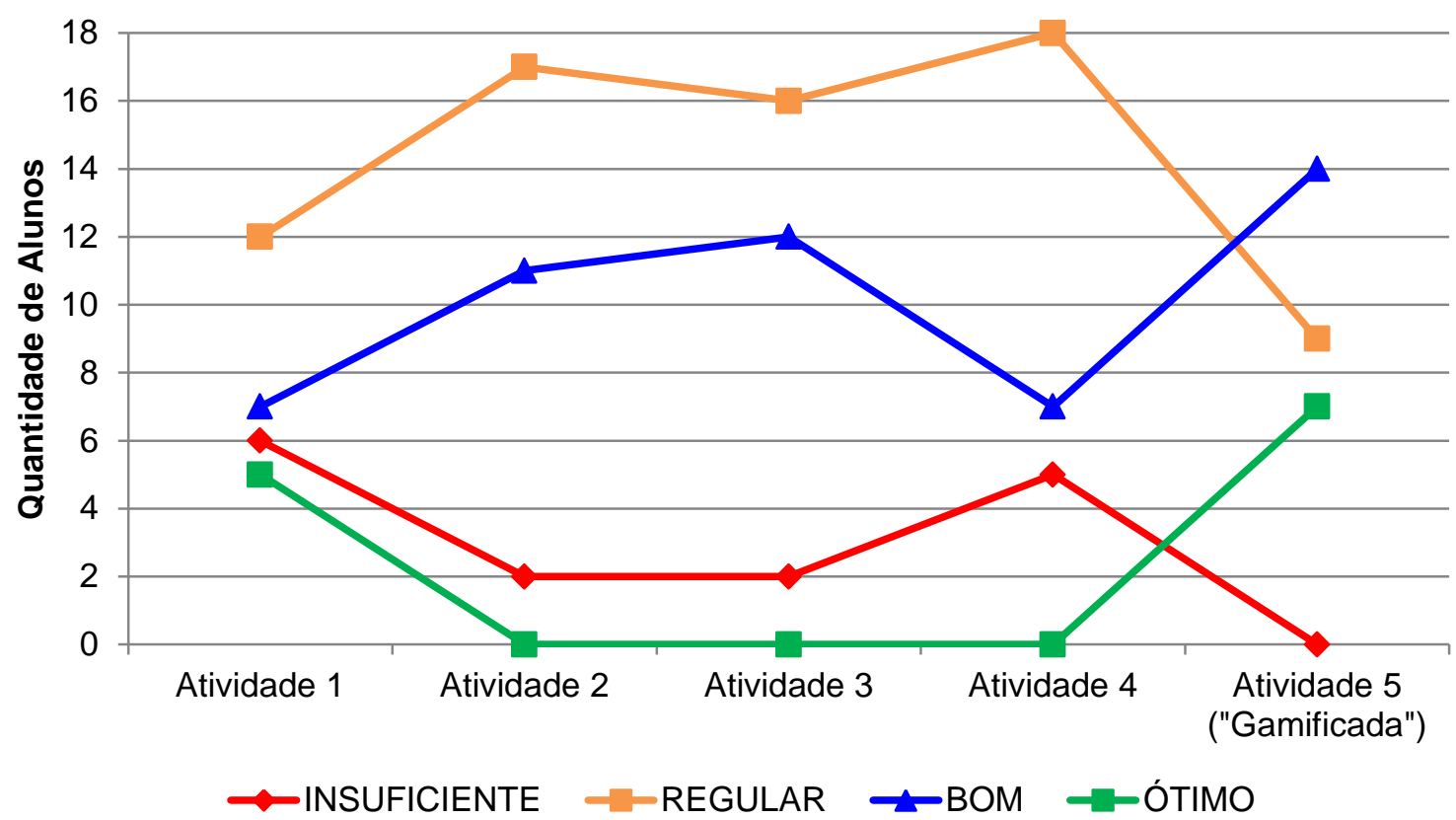

Figura 1. Desempenho da turma em atividades durante a disciplina DABD.

Apesar dos dados da Figura 1 não serem suficientes para indicar enfaticamente melhoria no desempenho de todos os alunos durante a base tecnológica (disciplina), é possível observar indícios disso ao visualizar uma queda em relação a conceitos como Insuficiente e Regular e, em contrapartida, um aumento de conceitos Bom e Ótimo.

O professor considerou que o baixo desempenho em atividades anteriores se deu devido à baixa motivação dos alunos em estudar os assuntos da base tecnológica (disciplina), além da escassa colaboração entre alunos nessas atividades. Alguns alunos acabam tendo mais facilidade em compreender certos conteúdos e, com um ambiente propício à colaboração, fica mais fácil a aquisição de conhecimento por parte dos demais alunos.

\section{Considerações Finais}

A realização da experiência relatada neste artigo propiciou, em geral, uma mudança de atitude dos alunos do curso técnico em informática em relação à disciplina durante as aulas "gamificadas" — os alunos, de modo geral, apresentaram-se participativos e mais entusiasmados com essas aulas em comparação com as aulas tradicionais.

É interessante, portanto, que os docentes reflitam sobre a possibilidade de adotarem mecanismos de gamificação para o ensino de pensamento computacional e programação, uma vez que é possível - fazendo-se uso de recursos encontrados em muitas salas de aula de cursos de computação - extrair resultados positivos para o processo de ensino-aprendizagem. 


\section{CBIE-LACLO 2015}

Anais dos Workshops do IV Congresso Brasileiro de Informática na Educação (CBIE 2015)

Apesar de ser uma experiência válida considerando a turma como um todo, nem todos os alunos mostraram-se "confortáveis" com toda a metodologia utilizada. Um dos alunos escolhidos de forma aleatória, por exemplo, estava literalmente tremendo de nervosismo por ir até a lousa responder a primeira etapa de um dos problemas. É interessante, portanto, considerar que os alunos possuem Estilos de Aprendizagem diferenciados [Aguiar, Fechine e Costa 2015b], sendo importante repensar nas estratégias utilizadas, tais como a formação aleatória dos grupos e a indicação aleatória para responder (talvez seja melhor uma indicação interna do grupo - mas é preciso avaliar cuidadosamente prós e contras, para evitar casos de alunos que se "acomodam" em parceria com colegas).

Com base nisso, almeja-se estimular reproduções similares dessa experiência e sugere-se também considerar o perfil de cada turma, identificando, por exemplo, características que podem propiciar a personalização do processo de ensinoaprendizagem em nível técnico e em demais níveis de ensino, tais como Traços de Personalidade [Aguiar, Fechine e Costa 2015a] e Estilos de Aprendizagem [Aguiar, Fechine e Costa 2015b].

Como trabalhos futuros, pretende-se replicar/expandir a experiência em outras turmas, extraindo uma avaliação por parte dos alunos sobre a metodologia.

\section{Agradecimentos}

À Escola Técnica Redentorista de Campina Grande - PB, especialmente à turma de alunos que participou desta experiência.

\section{Referências}

Aguiar, J. J. B.; Fechine, J. M.; Costa, E. (2015a). Identificando os Traços de Personalidade de Estudantes de um Curso Técnico em Informática. In: Anais do XXXV Congresso da Sociedade Brasileira de Computação (CSBC) - XXIII Workshop sobre Educação em Computação (WEI), Recife, sem paginação, 2015. Disponível em: <http://www.lbd.dcc.ufmg.br/colecoes/wei/2015/036.pdf>. Acesso em: 01 ago. 2015.

Aguiar, J. J. B.; Fechine, J. M.; Costa, E. (2015b). Utilização do Índice de Estilos de Aprendizagem de Felder-Soloman em Turmas de Nível Técnico, Graduação e PósGraduação em Computação. In: Anais do XXXV Congresso da Sociedade Brasileira de Computação (CSBC) - XXIII Workshop sobre Educação em Computação (WEI), Recife, sem paginação, 2015. Disponível em: $<$ http://www.lbd.dcc.ufmg.br/colecoes/wei/2015/035.pdf > . Acesso em: 01 ago. 2015.

Alves, G.; Warley, P.; Quadros, J.; Lignani, L.; Ogasawara, E. (2014). ControlHarvest: Ensino de Ecologia por Meio de Gamificação do Controle Biológico. In: Anais do XXV Simpósio Brasileiro de Informática na Educação (SBIE) — III Congresso Brasileiro de Informática na Educação (CBIE). Dourados, p. 342-351. Disponível em: <http://www.br-ie.org/pub/index.php/sbie/article/view/2960/2694>. Acesso em: 01 ago. 2015.

Borges, S. S.; Reis, H. M.; Durelli, V. H. S.; Bittencourt, I. I.; Jaques, P. A.; Isotani, S. (2013). Gamificação Aplicada à Educação: Um Mapeamento Sistemático. In: Anais do XXIV Simpósio Brasileiro de Informática na Educação (SBIE) - II Congresso 


\section{CBIE-LACLO 2015}

Anais dos Workshops do IV Congresso Brasileiro de Informática na Educação (CBIE 2015)

Brasileiro de Informática na Educação (CBIE). Campinas, p. 234-243. Disponível em: <http://www.br-ie.org/pub/index.php/sbie/article/view/2501/2160>. Acesso em: 01 ago. 2015.

Chaves, R. O.; Tavares, E. M. C.; Rodrigues, I. C.; Oliveira, S. R. B.; Favero, E. L. (2010). A Contribuição da Filosofia de Platão e de Aristóteles para o Ensino do Paradigma de Orientação a Objetos. In: Anais do XXI Simpósio Brasileiro de Informática na Educação (SBIE). João Pessoa, sem paginação. Disponível em: $<$ http://www.br-ie.org/pub/index.php/sbie/article/view/1520/1285>. Acesso em: 01 ago. 2015.

Da Silva, L. R. A.; De Queiroz, R. J. G. B. (2014). Aprendizagem baseada em jogos: Uma reflexão sobre o modelo de currículo da Quest to Learn. In: Anais do XX Workshop de Informática na Escola (WIE) - III Congresso Brasileiro de Informática na Educação (CBIE). Dourados, p. 86-90. Disponível em: $<$ http://www.br-ie.org/pub/index.php/wie/article/view/3086/2594>. Acesso em: 01 ago. 2015.

Domínguez, A.; Saenz-de-Navarrete, J.; De-Marcos, L.; Fernández-Sanz, L.; Pagés, C.; Martínez-Herráiz, J. (2013). Gamifying learning experiences: Practical implications and outcomes. Computers \& Education, vol. 63, p. 380-392.

Fernandes, A. M. R.; Castro, F. S. (2013). Ambiente de Ensino de Química Orgânica Baseado em Gamificação. In: Anais do XXIV Simpósio Brasileiro de Informática na Educação (SBIE) - II Congresso Brasileiro de Informática na Educação (CBIE). Campinas, p. 124-133. Disponível em: <http://www.brie.org/pub/index.php/sbie/article/view/2490/2149>. Acesso em: 20 ago. 2015.

Figueiredo, K. S. (2015). Proposta de Gamificação de Disciplinas em um Curso de Sistemas de informação. In: Anais do XI Simpósio Brasileiro de Sistemas de Informação (SBSI). Goiânia, p. 603-606. Disponível em: $<$ http://www.lbd.dcc.ufmg.br/colecoes/sbsi/2015/081.pdf>. Acesso em: 01 ago. 2015.

França, R. M.; Reategui, E. B. (2013). SMILE-BR: aplicação de conceitos de gamificação em um ambiente de aprendizagem baseado em questionamento. In: Anais do XXIV Simpósio Brasileiro de Informática na Educação (SBIE) — II Congresso Brasileiro de Informática na Educação (CBIE). Campinas, p. 366-375. Disponível em: <http://www.br-ie.org/pub/index.php/sbie/article/view/2515/2173>. Acesso em: 01 ago. 2015.

Haetinger, W.; Graziola Junior, P. G. (2002). Utilização de um carro-robô construído com componentes de baixo custo como ferramenta de apoio ao aprendizado dos conceitos de orientação a objetos. In: Anais do VI Congresso Iberoamericano de Informática Educativa. Vigo: Servicio de Publicacións da Universidade de Vigo, p. 1-4. Disponível em: <http://lsm.dei.uc.pt/ribie/docfiles/txt200373118616paper201.pdf $>$. Acesso em: 01 ago. 2015.

Kapp, K. M. (2012). The Gamification of Learning and Instruction: Game-Based Methods and Strategies for Training and Education. San Francisco: Pfeiffer. ISBN: 978-1-118-09634-5. 


\section{CBIE-LACLO 2015}

Anais dos Workshops do IV Congresso Brasileiro de Informática na Educação (CBIE 2015)

Lee, J. J.; Hammer, J. (2011). Gamification in Education: What, How, Why Bother?. Academic Exchange Quarterly, 15(2), p. 1-5. Disponível em: $<$ https://www.uwstout.edu/soe/profdev/resources/upload/Lee-Hammer-AEQ2011.pdf $>$. Acesso em: 01 ago. 2015.

Medeiros, C. D.; Fernandes, A. M.; Damasceno, E. F. (2014). Uma Abordagem Gamificada para Prevenção do uso de Drogas com Adolescentes. In: Anais do XXV Simpósio Brasileiro de Informática na Educação (SBIE) - III Congresso Brasileiro de Informática na Educação (CBIE). Dourados, p. 1173-1177. Disponível em: $<$ http://www.br-ie.org/pub/index.php/sbie/article/view/3063/2571>. Acesso em: 01 ago. 2015.

Paschoal, L.; Bento, T.; Velasco, T.; Schocair, C. O.; Castaneda, R.; Oliveira, T.; Ogasawara, E. (2014). JOE: Jogo Ortográfico Educacional. In: Anais do XXV Simpósio Brasileiro de Informática na Educação (SBIE) - III Congresso Brasileiro de Informática na Educação (CBIE). Dourados, p. 652-656. Disponível em: $<$ http://www.br-ie.org/pub/index.php/sbie/article/view/2995/2506>. Acesso em: 01 ago. 2015.

Scaico, P. D.; De Queiroz, R. J. G. B. (2013). A educação do futuro: uma reflexão sobre aprendizagem na era digital. In: Anais do XXIV Simpósio Brasileiro de Informática na Educação (SBIE) - II Congresso Brasileiro de Informática na Educação (CBIE). Campinas, p. 889-893. Disponível em: <http://www.brie.org/pub/index.php/sbie/article/view/2567/2225>. Acesso em: 01 ago. 2015.

Seixas, L. R.; Gomes, A. S.; Melo Filho, I. J.; Rodrigues, R. L. (2014). Gamificação como Estratégia no Engajamento de Estudantes do Ensino Fundamental. In: Anais do XXV Simpósio Brasileiro de Informática na Educação (SBIE) - III Congresso Brasileiro de Informática na Educação (CBIE). Dourados, p. 559-568. Disponível em: <http://www.br-ie.org/pub/index.php/sbie/article/view/2985/2496>. Acesso em: 01 ago. 2015.

Vahldick, A. (2007). Uma experiência lúdica no ensino de programação orientada a objetos. In: Anais do XVIII Simpósio Brasileiro de Informática na Educação (SBIE). São Paulo, p. 1-8. Disponível em: <https://2936dfd1-a-62cb3a1a-ssites.googlegroups.com/site/adilsonv77/36388.pdf>. Acesso em: 01 ago. 2015.

Zaina, L. A. M.; Caversan, F. L. (2005). Projeto Multidisciplinar: uma Experiência Prática no Ensino de Programação em um Curso de Engenharia da Computação. In: Anais do XXXIII Congresso Brasileiro de Ensino de Engenharia (COBENGE). Campina Grande, sem paginação. Disponível em: $<$ http://www.abenge.org.br/CobengeAnteriores/2005/artigos/SP-15-285453218051118683317399.pdf>. Acesso em: 01 ago. 2015. 